\title{
Interleukin-31 is overexpressed in skin and serum in cutaneous T-cell lymphomas but does not correlate to pruritus
}

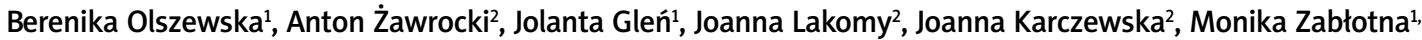 \\ Marta Malek ${ }^{1}$, Jerzy Jankau ${ }^{3}$, Magdalena Lange ${ }^{1}$, Wojciech Biernat ${ }^{2}$, Roman J. Nowicki' ${ }^{1}$, Małgorzata Sokołowska-Wojdyło ${ }^{1}$
}

${ }^{1}$ Department of Dermatology, Venereology and Allergology, Medical University of Gdansk, Gdansk, Poland

${ }^{2}$ Department of Pathology, Medical University of Gdansk, Gdansk, Poland

${ }^{3}$ Department of Plastic Surgery, Medical University of Gdansk, Gdansk, Poland

Adv Dermatol Allergol 2022; XXXIX (1): 81-87

DOI: https://doi.org/10.5114/ada.2020.100824

\begin{abstract}
Introduction: Cutaneous T-cell lymphomas (CTCL) are malignant lymphoproliferative disorders accompanied by persistent pruritus. Pruritogenic role of interleukin-31 (IL-31) has been studied extensively and was proven in atopic dermatitis (AD), while its role in CTCL is still rather vague.

Aim: To investigate IL-31 serum level along with IL-31, IL-31 receptor $\alpha$ (IL-31RA) and oncostatin M receptor $\beta$ (OSMR) skin expression in CTCL and compare it to controls: $A D$ and healthy volunteers.

Material and methods: The level of IL-31 in serum was measured using ELISA, while IL-31 and receptors' expression in the skin were measured using immunohistochemistry and correlated with the stage of disease and pruritus severity.

Results: Expression of IL-31 and IL-31 receptor in serum and skin were significantly higher in CTCL and AD in comparison to healthy controls. No significant correlation between the IL-31 serum level and pruritus severity in CTCL patients was found. There was also no correlation between IL-31/IL-31RA/OSMR expression in the skin and CTCL pruritus, while IL-31 and IL-31RA in CTCL skin negatively correlated with the stage of disease.

Conclusions: Our data indicate that IL-31 does not play a crucial role in pruritus in CTCL but it is rather involved in the pathogenesis of the disease. It seems that IL-31 plays an essential role in the pruritus pathomechanism that is unique to $A D$.
\end{abstract}

Key words: cutaneous T-cell lymphomas, interleukin-31, oncostatin M receptor $\beta$, IL-31RA, pruritus, atopic dermatitis.

\section{Introduction}

Cutaneous T-cell lymphomas (CTCLS) comprise a clinically heterogeneous group of malignant lymphoproliferative disorders. Most common subtypes are mycosis fungoides (MF) and Sezary syndrome (SS), an aggressive variant of CTCL [1]. Pruritus is one of the hallmarks of CTCL, it affects $66 \%$ to $88 \%$ of patients, significantly reduces quality of life and responds poorly to anti-histamine medications [2-4]. The mechanism of underlying CTCL pruritus is complex and not fully elucidated, just as the pathogenesis of the disease. However, emerging evidence indicates that the cytokine milieu of the skin might play a crucial role.

Interleukin- 31 (IL-31) is a four-helix bundle cytokine that belongs to IL-6 cytokine family. IL-31 is produced by a variety of cells, predominantly by CLA+ CD45RO+ memory $T$ cells and signals through a heterodimeric receptor consisting of IL-31 RA and oncostatin M receptor $\beta$ (OSMR) [5-8]. As IL-31 RA and OSMR are expressed in dorsal root ganglia and epidermis, IL-31 is indicated to be a link between the neural and immune system in pruritus mechanism [9]. A series of research on transgenic mice demonstrated that overexpression of IL-31 elicited atopic-like dermatitis, a cutaneous injection resulted in scratching behaviour, while anti-IL-31 antibody reduced itch in mice [10-13]. Moreover, the IL-31 level has been shown to be elevated in pruritic dermatoses such as AD and to correlate with itch intensity and disease severity [14-17]. Recently, an anti-IL-31 receptor antibody (Nemolizumab) was shown to significantly improve pruritus in patients with atopic dermatitis [18, 19].

Address for correspondence: Berenika Olszewska, Department of Dermatology, Venereology and Allergology, Medical University of Gdansk, Gdansk, Poland, phone: +48 5858440 10, e-mail: berenika.olszewska@o2.pl Received: 29.09.2020, accepted: 8.10.2020. 


\section{Aim}

Several studies concerning IL-31 expression in CTCL have been published, however, its role in CTCL is still unclear. Given the vague association between IL-31 and progression of the disease and pruritus severity, we examined the expression of IL-31 and its receptor in CTCL and control groups (AD patients and healthy controls) and correlation with pruritus and the stage of CTCL.

\section{Material and methods}

The study group included 42 patients diagnosed with CTCL (38 MF, 4 SS, 24 males, 18 females, median age 64.5, age range: 26-90) according to the WHO-EORTC classification for primary cutaneous lymphomas [1]. Patients with CTCL were sub-grouped into stages: la (10), Ib (10), IIa (1), IIb (9), III (6), IV (6) according to the TNMB staging system [20]. Due to a relatively scarce study group we also divided CTCL patients into the early-stage (IA-IIA: 21) and advanced-stage group (IIB-IV: 21).

The control group consisted of 20 patients diagnosed with $\mathrm{AD}$ (males 14, females 6, median age: 32, age range: 21-63) and 24 healthy individuals (5 males, 19 females, median age 37.5 , age range: $18-73$ ) without any history of pruritus, $A D, C T C L$ or other immune diseases. Adult patients with moderate (11) and severe (9) AD were as sessed by the scoring atopic dermatitis index (SCORAD) and those who fulfilled the Hanifin and Rajka criteria were enrolled in the study. The severity of pruritus was assessed according to the Visual Analog Scale (VAS) and Numeric Rating Scale (NRS) in CTCL and AD patients (VAS/NRS: $0=$ no pruritus, $>0-3$ points $=$ mild pruritus, $\geq 3-7$ points $=$ moderate pruritus, $\geq 7-10$ points $=$ severe pruritus).

The study was approved by the Ethic Committee of the Medical University of Gdańsk and was conducted according to the principles of the Declaration of Helsinki. All participants signed informed consent prior to any study procedure. Serum samples of CTCL and AD patients and healthy individuals were obtained and afterwards stored frozen. Punch biopsies were taken from the lesional skin of $A D$ and $C T C L$ patients but also from the normal skin of healthy individuals.

\section{Immunohistochemistry}

Expression of IL-31, IL-31 RA and OSMR in the skin was determined by the immunohistochemistry (IHC) performed on paraffin-embedded skin samples obtained from patients with $C T C L$ and $A D$, and healthy controls. Primary antibody combinations were anti-IL-31 (rabbit polyclonal antibody, dilution 1 : 25, orb157687; Biorbyt, Cambridge, United Kingdom), anti-IL-31 RA (rabbit polyclonal antibody, dilution 1 : 100, GTX54294; GeneTex, CA, U.S.A.) and anti-OSMR polyclonal antibodies (rabbit polyclonal antibody, dilution 1 : 50, orb247562; Biorbyt,
Cambridge, United Kingdom). Slides were incubated with primary anti-IL-31, anti-IL-31 RA and anti-OSMR antibodies overnight at $4^{\circ} \mathrm{C}$. As a secondary antibody, the Novolink Polymer Detection System from Novocastra was used. All slides were counterstained with Haematoxylin. The negative controls were sections treated without any primary antibody. The expression of IL-31, IL-31RA, OSMR in epidermis and in the dermal infiltrate was evaluated using a light microscope Olympus BX40 by pathologists who were blinded to the patients' clinical data.

The immunoreactivity of IL-31, IL-31RA and OSMR antibodies demonstrated homogeneous weak positive staining throughout epidermis and skin appendages from healthy controls and it did not vary much between antigens. The horny cell layer was not stained by the antibodies. All CTCL and AD samples presented positive IL-31, IL-31RA and OSMR staining. Immunoreactivity of IL-31 and IL-31 receptor was observed both in epidermis and lymphocytic infiltrate. IL-31 staining showed predominantly cytoplasmic pattern, IL-31-RA - cytoplasmic pattern and OSMR - cytoplasmic-nuclear pattern. The horny cell layers were not stained by the IL-31, IL-31 RA and OSMR antibodies. Grading of immunoreactivity was performed both in epidermis and lymphoid dermal infiltrate separately.

\section{Semiquantitative analysis}

We used the histoscore method (HSCORE-index) which is a semiquantitative analysis applied in immunohistochemistry assessment [21]. The total field of every section was examined by evaluating the percentage of positive cells and the intensity of staining (no immunoexpression $=0$, weak $=+1$, moderate $=+2$, strong $=+3$ ) by two independent investigators. The evaluation was compared and any conflicting results were re-examined. The HSCORE was obtained by the formula: $1 \times$ (percentage of weakly stained cells) $+2 \times$ (percentage of moderately stained cells) $+3 \times$ (percentage of strongly stained cells) (range: $0-300)$.

\section{Enzyme-linked immunosorbent assay methodology (ELISA)}

Serum IL-31 levels were measured using ELISA standard kit (BioVendor-Laboratorni medicina a.s., Brno, Czech Republic). This product has been tested by Quality Control and complied with internal specifications. We analysed 42 CTCL, 20 control group serum samples and 18 AD serum samples with an IL-31 ELISA. All procedures were performed according to the manufacturer's protocol.

\section{Statistical analysis}

For evaluation of normality and equality of variances, Shapiro-Wilk test and Levene's tests were used. As the requirements of the tests were not met, statistical analysis was carried out using the Mann-Whitney $U$ test and 
Table 1. The median and range of IL-31 expression in serum and IL-31, IL-31 RA and OSMR HSCORE levels in the epidermis and dermis of CTCL, AD and CG

\begin{tabular}{llccccc}
\hline $\begin{array}{l}\text { Skin expression, } \\
\text { H score median (range) }\end{array}$ & CTCL & $\begin{array}{c}\text { CTCL } \\
\text { early stage }\end{array}$ & $\begin{array}{c}\text { CTCL advanced } \\
\text { stage }\end{array}$ & AD & CG \\
\hline IL-31 & Epidermis & $200(100-300)$ & $200(100-300)$ & $200(100-300)$ & $100(100-200)$ & 100 \\
\cline { 2 - 7 } & Dermis & $115(35-280)$ & $130(45-200)$ & $110(35-280)$ & $85(10-165)$ & $*$ \\
\hline \multirow{2}{*}{ IL-31RA } & Epidermis & $100(100-200)$ & $200(100-200)$ & $100(100-200)$ & $150(100-200)$ & 100 \\
\cline { 2 - 7 } & Dermis & $100(20-280)$ & $130(30-215)$ & $80(20-280)$ & $162(40-200)$ & $*$ \\
\hline OSMR & Epidermis & $100(100-200)$ & $100(100-200)$ & $100(100-200)$ & $100(100-200)$ & 100 \\
\cline { 2 - 7 } & Dermis & $100(5-200)$ & $100(50-195)$ & $100(5-200)$ & $95(30-215)$ & $*$ \\
\hline IL-31 serum expression, & 4.82 & 4.89 & 4.62 & 6.09 & 0.00 \\
median (range) $[\mathrm{pg} / \mathrm{ml})$ & $(2.89-7.74)$ & $(3.34-7.74)$ & $(2.89-6.27)$ & $(4.27-17.48)$ & $(0.00-2.73)$ \\
\hline
\end{tabular}

CTCL - cutaneous T-cell lymphomas, AD - atopic dermatitis, CG - healthy control group, *dermis was not examined in the healthy control group due to lack of inflammatory infiltrate.

Kruskal-Wallis one-way analysis of variance (ANOVA). Furthermore, the $\chi^{2}$ Pearson test and the Spearman correlation test were performed. Statistical analyses were performed using Statistica 12 . The results were considered statistically significant when $p<0.05$.

\section{Results}

\section{Pruritus characteristics}

Pruritus was observed in 32 out of 42 (76.2\%) CTCL patients and in all patients with AD (100\%). Significantly more patients with $A D$ experienced pruritus than patients with CTCL $(p=0.023)$. The median severity of pruritus in patients with CTCL according to NRS was 3.0 and VAS was 3.3 (range: 0-10). In AD patients the pruritus score was 6.0 (NRS) and 6.4 (VAS) (range: 0-10). There was a statistically significant difference in pruritus severity between AD and CTCL patients (NRS $p=0.022$; VAS $p=0.009$ ). Pruritus was more severe in advanced (NRS/ VAS 5) than early-stage CTCL (NRS/VAS 3) but the difference was not statistically significant (NRS $p=0.907$; $\operatorname{VAS} p=0.539)$.

\section{Serum IL-31}

The serum IL-31 level in CTCL, AD and healthy control group is summarized in Table 1 . Statistical analysis revealed significant differences in the serum IL-31 level between $C T C L, A D$ and healthy control group (Figure 1). Serum IL-31 levels in AD and CTCL patients were significantly higher than in healthy controls $(p<0.001)$. Moreover, the IL-31 serum level was significantly higher in AD patients than $C T C L$ patients ( $p=0.001$ ).

We have also found that serum IL-31 level was significantly higher in $A D$ patients than patients with early-stage CTCL ( $p=0.011)$ and also advanced-stage CTCL $(p=0.001)$. On the other hand, there were no statistically significant differences in the IL-31 serum level between advanced- and early-stage CTCL ( $p=0.343)$, and also pruritic and non-pruritic CTCL (VAS $p=0.421$; NRS $p=0.805$ ).

\section{Serum IL-31 in relation to pruritus and stage of CTCL}

We did not find any correlation between serum IL-31 levels and pruritus intensity according to NRS/ VAS ratings in patients with CTCL $(r=0.012 ; p=0.940$; $r=-0.007 ; p=0.960$, respectively). On the other hand, pruritus severity according to NRS and VAS ratings significantly correlated with the IL-31 serum level in AD patients (NRS $r=0.47 ; p=0.044$; VAS $r=0.48 ; p=$ 0.042 ). We also did not find any significant correlation

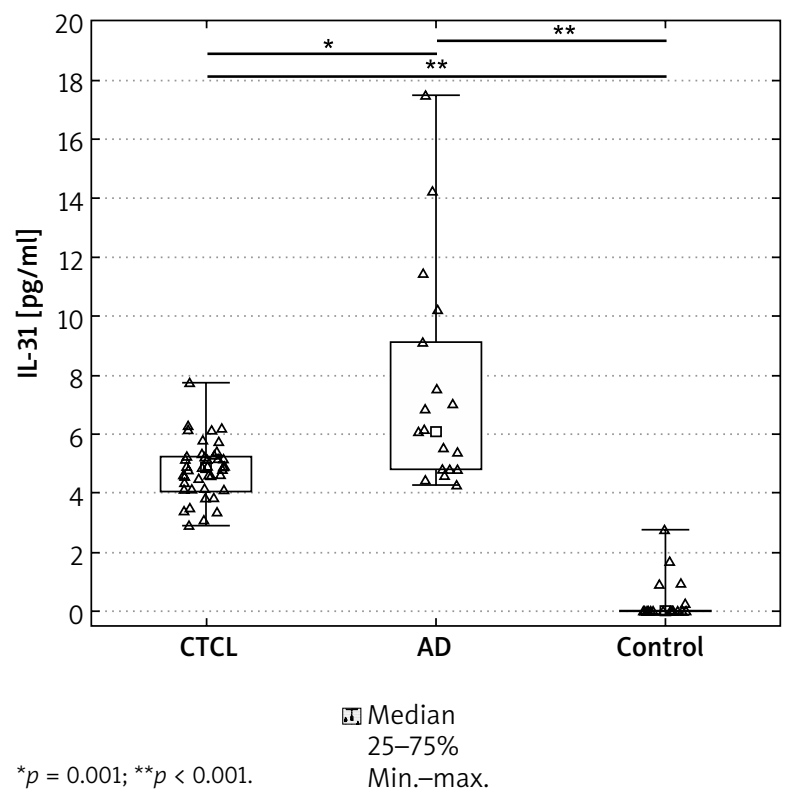

Figure 1. Serum interleukin-31 (IL-31) level in patients with cutaneous T-cell lymphoma (CTCL), atopic dermatitis (AD) and healthy control group $(p<0.001)$. Asterisks indicate differences between CTCL, AD and healthy control group 
IL-31
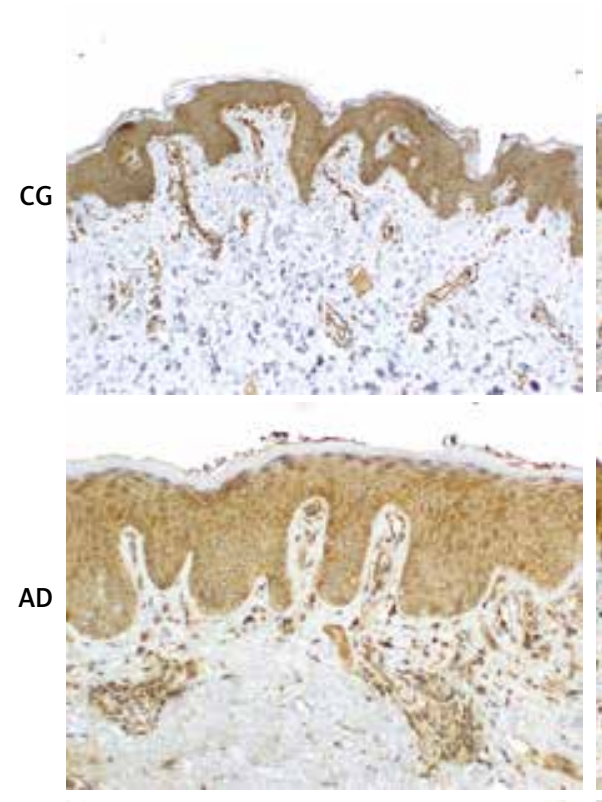

CTCL
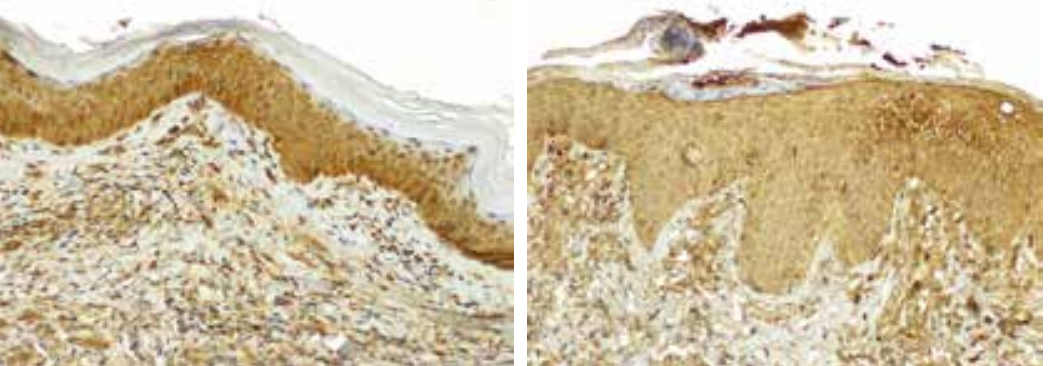

IL-31RA

OSMR
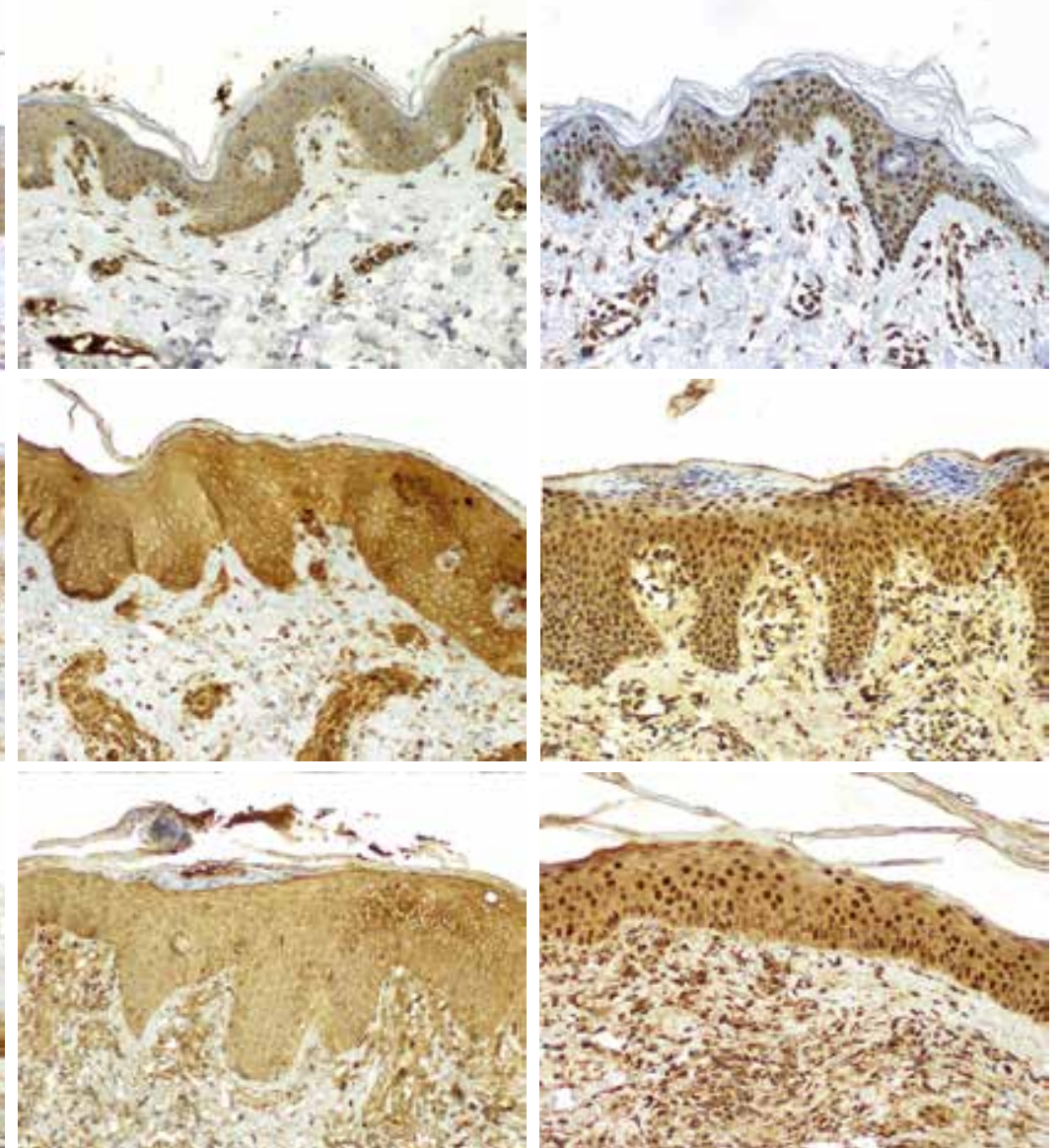

Figure 2. Immunohistochemical staining for IL-31, IL-31 receptor $\alpha$ (IL-31RA) and oncostatin M receptor $\beta$ (OSMR). Immunohistochemical staining in healthy skin of the control group (CG) showed IL-31 and IL-31RA cytoplasmic pattern limited to epidermis and skin appendages while OSMR showed cytoplasmic-nuclear staining. Epidermis and dermal infiltrate of the lesional skin of cutaneous T-cell lymphoma (CTCL - early stage MF) and atopic dermatitis (AD) showed cytoplasmic pattern of IL-31 and IL-31RA while OSMR showed cytoplasmic-nuclear pattern (DAB staining, magnification 20x)

between IL-31 serum levels and the CTCL stage $(r=-0.10$; $p=0.527)$.

\section{Expression of IL-31, IL-31 RA, OSMR in the skin}

Representative images of IL-31, IL-31 RA and OSMR expression in CTCL, AD and healthy skin are demonstrated in Figure 2. The HSCORE level (median and range) of IL-31, IL-31 RA and OSMR expression in the epidermis and dermis are presented in Table 1.

Statistical analysis revealed significant differences in IL-31, IL-31 RA and OSMR HSCORE between CTCL, AD and healthy control group. Both $C T C L$ and $A D$ groups presented a significantly higher expression of IL-31, IL-31RA and OSMR in epidermis in comparison to healthy controls (CTCL/AD: $p=0.038 / p<0.001 ; p<0.001 / p=0.004$; $p=0.016 / p=0.010$; respectively). The IL-31 HSCORE level was significantly higher in lymphocytic infiltrate in the dermis of CTCL skin samples ( $p=0.001)$ whereas IL-31RA was significantly overexpressed in lymphocytic infiltrate of AD skin samples $(p=0.020)$. There were no significant differences in OSMR expression in epidermis and dermis between $C T C L$ and AD skin samples.

A statistically significant difference was found between AD and early-stage CTCL samples in the HSCORE IL-31 level both in epidermis $(p=0.037)$ and dermis $(p<0.001)$. There was also a statistically significant difference between AD and advanced-stage CTCL samples in HSCORE IL-31 ( $p=0.037)$ and IL-31RA $(p=0.006)$ levels in dermis. Only immunoreactivity staining for IL-31RA in epidermis differed significantly between the early and advanced stage of CTCL $(p=0.034)$.

\section{Skin expression of IL-31 and receptors in relation to CTCL pruritus}

No correlation was found between both epidermal and dermal IL-31, IL-31 receptor subunit levels and severity of pruritus according to NRS and VAS ratings in $\mathrm{CTCL}$ patients. 


\section{Skin expression of IL-31 and receptors in relation to the stage of CTCL}

The CTCL stage negatively correlated with IL-31 levels in dermal infiltrate $(r=-0.31 ; p=0.047)$, but not with the epidermal IL-31 level $(r=-0.26 ; p=0.100)$. The epidermal IL-31RA expression negatively correlated with the CTCL stage ( $r=-0.31 ; p=0.043)$, while the IL-31RA dermal expression correlation was not significant $(r=-0.27$; $p=0.081)$. OSMR expression both in epidermis and dermis did not correlate with the stage of disease $(p=0.998 ; p=0.823$ respectively).

\section{Discussion}

The role of IL-31 and its receptor components (IL-31 RA and OSMR) in pruritus that accompanies AD has been determined by many studies [8, 10, 14, 15, 22] while reports on IL-31's role in CTCL are scarce and conclusions are rather vague. Several recent studies have shown increased serum levels of IL-31 in CTCL patients [22-26] while there is only one study that has investigated the expression of IL-31 and its receptors in the skin from CTCL patients [27]. Our study is the first one that compares the expression of IL-31 and its receptors in the skin and serum of CTCL and AD patients and healthy controls.

Similarly to previous studies, we have found that pruritus was more severe in the advanced than the early stage of CTCL [26]. Moreover, the mean pruritus score was significantly higher in $A D$ than $C T C L$ and it occurred in all AD patients versus $76 \%$ of CTCL patients.

We observed that the IL-31 serum level in CTCL and $A D$ patients was significantly higher than in the control group, which is in line with all previous reports [15, 23-26]. However, in our study the IL-31 serum level did not reflect the severity of CTCL. Lack of correlation between the IL-31 serum level and the stage of CTCL in the presence of a significantly higher serum level compared to the control group suggests its role in the pathogenesis of the disease, however, it seems not to contribute to disease progression.

The pruritogenic role of IL-31 in CTCL remains unclear. Several serum studies documented either correlation between IL-31 mRNA and itch intensity [23] or a higher but not significant IL-31 serum level in severely pruritic than non-pruritic CTCL patients [25]. The recent study involving the largest MF group (62 MF patients) by Malek et al. did not find such correlations, indicating that IL-31 is rather involved in pathogenesis of CTCL [26]. Our investigations also showed no difference in the IL-31 serum level between pruritic and non-pruritic CTCL patients and no correlation between the serum IL-31 concentration and pruritus severity in the CTCL group. Contrary to Nattkemper et al. [27] study, we did not find any correlation between both IL-31 and IL-31 receptor in skin and pruritus intensity in CTCL patients, suggesting that IL-31 is not involved in the pathomechanism of pruritus in CTCL. Meng et al. reported that IL-31 enhanced synthesis of brain-derived natriuretic peptide, an itch mediator, in skin and DRG in AD [28]. Moreover, Hawro et al. [29] demonstrated that intradermal exposure to IL-31 does not induce an immediate itch in AD patients and healthy controls, it is possible that IL-31 could act indirectly by inducing other cells to release secondary itch mediators. We have also verified the hypothesis made by Nobbe et al. stating that the pruritogenic role of IL-31 is rather unique to $A D$ as compared to pruritic skin diseases in general [22]. We observed that the IL-31 serum level was significantly overexpressed in AD patients than in $\mathrm{CTCL}$ patients, which has not been reported yet. Moreover, we found a significant correlation between the IL-31 serum level and pruritus severity in AD patients. Those results indicate that IL-31 might actually play a central role in the pathogenesis of pruritus particularly in $A D$ rather than in other pruritic skin diseases. Further studies including a wider range of pruritic skin diseases would be required to compare IL-31 serum and skin levels to confirm those results.

Our study has found expressions and patterns of IL-31, IL-31RA and OSMR in epidermis and dermal infiltrate of both CTCL and AD tissue samples, which are in line with previous findings $[22,27]$. We demonstrated increased immunoreactivity of the IL-31 and IL-31 receptor components in $\mathrm{AD}$ and $\mathrm{CTCL}$ skin compared to healthy skin samples [8, 14, 22]. In contrast to Nattkemper et al. [27] study, which reported an increased expression of IL-31 receptor components limited to CTCL epidermis, we have observed overexpression also in the dermis. Interestingly, we found a significantly higher expression of IL-31 in dermal infiltrate in CTCL than AD skin samples, whereby it has not been reported yet. Unlike our findings, Nobbe et al. study showed increased immunoreactivity in AD subjects compared to other Th-2 weighted skin diseases including MF [22]. The discrepancy between findings may be explained by small study groups (AD and MF $n=5$ each) and the stage of disease [22]. It could be speculated that CTCL tumour cells together with inflammatory cells might be the source of the increased level of IL-31, however, the ability of malignant lymphocytes to produce IL-31 is a point of contention according to research results and it needs further studies [23, 25]. However, there are also several limitations of our study that should be considered. First, the disparity in the number of samples from female and male volunteers between studied groups. Second, the disparity in age between $C T C L$ and control groups (AD and healthy volunteers) that could be explained by the biological variables between diseases. Therefore, it would be beneficial to perform further studies that would include a suitable number of patients matched for sex and age.

Finally, our study showed a negative correlation between the CTCL stage and IL-31 expression limited to dermal infiltrate. IL-31 RA expression in epidermis correlated negatively with the CTCL stage as well. These findings 
should be interpreted with caution, however they seem to be particularly interesting as Th2 lymphocytes are the main source of IL-31 and the inflammatory environment becomes Th2-biased during the clinical course of CTCL [30]. The reason for declining expression of IL-31RA in epidermis in late-stage CTCL might be the lack of Th1 cytokines, mainly IFN- $\gamma$, which induce the expression of IL-31 RA in dendritic cells, monocytes and macrophages localized in epidermis [10]. IL-31 is expressed predominantly but not specifically by Th2 lymphocytes. However, Th1 cells, monocytes, macrophages and dendritic cells were also demonstrated to be the IL-31 source [5-7]. When it comes to malignant T cells in CTCL that possess a Th2-helper cell phenotype, Mobs et al. demonstrated that tumour cells are unlikely to be the source of IL-31 in CTCL [25]. Moreover, it was suggested that IL-31 expression does not result from a Th1 to Th2 conversion, but its upregulation is rather dependent on skin environment and stimulating factors such as IL-2, IL-4, and IL-13 [25, 31, 32]. It can be speculated that the dense dermal infiltrate of malignant T cells in advanced-stage CTCL skin suppresses the benign T-cells and bystander cells which are the source of IL-31, resulting in downregulation of IL-31.

\section{Conclusions}

Patients with $C T C L$ and $A D$ presented significantly higher IL-31 serum level IL-31, OSMR, IL-31RA skin expression in comparison to healthy controls. Overexpression of IL-31 and its receptor in CTCL compared to healthy controls suggest involvement in the CTCL pathogenesis. Moreover, our data indicate that IL-31 is not involved in the pathogenesis of pruritus in CTCL. Our findings suggest that IL-31 plays a central role in the pruritus pathomechanism that is specific to $A D$ rather than pruritic dermatoses in general, yet this issue requires further studies.

\section{Acknowledgments}

The study was supported by the Polish Ministry of Science and Higher Education Grant. Project No. ST 02$0066 / 07 / 253$.

\section{Conflict of interest}

The authors declare no conflict of interest.

\section{References}

1. Willemze R, Cerroni L, Kempf W, et al. The 2018 update of the WHO-EORTC classification for primary cutaneous lymphomas. Blood 2019; 133: 1703-14.

2. Wright A, Wijeratne A, Hung T, et al. Prevalence and severity of pruritus and quality of life in patients with cutaneous T-cell lymphoma. J Pain Symptom Manage 2013; 45: 114-9.

3. Demierre MF, Gan S, Jones J, Miller DR. Significant impact of cutaneous T-cell lymphoma on patients' quality of life: results of a 2005 National Cutaneous Lymphoma Foundation Survey. Cancer 2006; 107: 2504-11.

4. Vij A, Duvic M. Prevalence and severity of pruritus in cutaneous T cell lymphoma. Int J Dermatol 2012; 51: 930-4.

5. Hermanns HM. Oncostatin M and interleukin-31: cytokines, receptors, signal transduction and physiology. Cytokine Growth Factor Rev 2015; 26: 545-58.

6. Cornelissen C, Lüscher-Firzlaff J, Baron JM, Lüscher B. Signaling by IL-31 and functional consequences. Eur I Cell Biol 2012; 91: 552-66.

7. Zhang Q, Putheti P, Zhou Q, et al. Structures and biological functions of IL-31 and IL-31 receptors. Cytokine Growth Factor Rev 2008; 19: 347-56.

8. Bilsborough J, Leung DY, Maurer M, et al. IL-31 is associated with cutaneous lymphocyte antigen-positive skin homing T cells in patients with atopic dermatitis. J. Allergy Clin Immunol 2006; 117: 418-25.

9. Cevikbas F, Wang X, Akiyama T, et al. A sensory neuronexpressed IL-3q receptor mediates Thelper cell- dependent itch: involvement of TRPV1 and TRPA1. J Allergy Clin Immunol 2014; 133: 448-60.

10. Dillon SR, Sprecher C, Hammond A, et al. Interleukin 31, a cytokine produced by activated T cells, induces dermatitis in mice. Nat Immunol 2004; 5: 752-60.

11. Grimstad O, Sawanobori Y, Vestergaard C, et al. Anti-interleukin-31 antibodies ameliorate scratching behaviour in NC/ Nga mice: a model of atopic dermatitis. Exp Dermatol 2009; 18: 35-43.

12. Arai I, Tsuji M, Miyagawa K, et al. Repeated administration of IL-31 upregulates IL-31 receptor A (IL-31RA) in dorsal root ganglia and causes severe itch-associated scratching behaviour in mice. Exp Dermatol 2015; 24: 75-8.

13. Kasutani K, Fujii E, Ohyama S, et al. Anti-IL-31 receptor antibody is shown to be a potential therapeutic option for treating itch and dermatitis in mice. Br J Pharmacol 2014; 171 : 5049-58.

14. Sonkoly E, Muller A, Lauerma Al, et al. IL-31: a new link between T cells and pruritus in atopic skin inflammation. J Allergy Clin Immunol 2006; 117: 411-7.

15. Raap U, Wichmann K, Bruder M, et al. Correlation of IL-31 serum levels with severity of atopic dermatitis. J Allergy Clin Immunol 2008; 122: 421-3.

16. Ferretti E, Tripodo C, Pagnan G, et al. The interleukin (IL)-31/ IL-31R axis contributes to tumor growth in human follicular lymphoma. Leukemia 2015; 29: 958-67.

17. Kim S, Kim HJ, Yang HS, et al. IL-31 serum protein and tissue mRNA levels in patients with atopic dermatitis. Ann Dermatol 2011; 23: 468-73.

18. Nemoto O, Furue M, Nakagawa $\mathrm{H}$, et al. The first trial of CIM331, a humanized antihuman interleukin-31 receptor A antibody, in healthy volunteers and patients with atopic dermatitis to evaluate safety, tolerability and pharmacokinetics of a single dose in a randomized, double-blind, placebo-controlled study. Br I Dermatol 2016; 174: 296-304.

19. Ruzicka, T, Hanifin JM, Furue M, et al. Anti-interleukin-31 receptor A antibody for atopic dermatitis. N Engl J Med 2017; 376: 826-35.

20. Olsen E, Vonderheid E, Pimpinelli N, et al. Revisions to the staging and classification of mycosis fungoides and Sezary syndrome: a proposal of the International Society for Cutaneous Lymphomas (ISCL) and the cutaneous lymphoma task force of the European Organization of Research and Treatment of Cancer (EORTC). Blood 2007; 110: 1713-22. 
21. Fedchenko N, Reifenrath J. Different approaches for interpretation and reporting of immunohistochemistry analysis results in the bone tissue - a review. Diagn Pathol 2014; 9: 221.

22. Nobbe S, Dziunycz P, Mühleisen B, et al. IL-31 expression by inflammatory cells is preferentially elevated in atopic dermatitis. Acta Derm Venereol 2012; 92: 24-8.

23. Singer EM, Shin DB, Nattkemper LA, et al. IL-31 is produced by the malignant T-cell population in cutaneous T-cell lymphoma and correlates with CTCL pruritus. J Invest Dermatol 2013; 133: 2783-5.

24. Ohmatsu H, Sugaya M, Suga H, et al. Serum IL-31 levels are increased in patients with cutaneous T-cell lymphoma. Acta Derm Venereol 2012; 92: 282-3.

25. Mobs M, Gryzik S, Haidar A, et al. Analysis of the IL-31 pathway in mycosis fungoides and Sezary syndrome. Arch Dermatol Res 2015; 307: 479-85.

26. Malek M, Gleń J, Rębała K, et al. IL-31does no correlate to pruritus related to early stage cutaneous T-cell lymphomas but is involved in pathogenesis of the disease. Acta Derm Venereol 2015; 95: 283-8.

27. Nattkemper LA, Martinez-Escala ME, Gelman AB, et al. Cutaneous T-cell lymphoma and pruritus: the expresion of IL-31 and its receptors in the skin. Acta Derm Venereol 2016; 96 : 894-8.

28. Meng J, Moriyama M, Feld M, et al. New mechanism underlying IL-31-induced atopic dermatitis. J Allergy Clin Immunol 2018; 141: 1677-89.

29. Hawro T, Saluja R, Weller K, et al. Interleukin-31 does not induce immediate itch in atopic dermatitis patients and healthy controls after skin challenge. Allergy 2014; 69: 113-7.

30. Bağci IS, Ruzicka T. IL-31: a new key player in dermatology and beyond. J Allergy Clin Immun 2018; 141: 858-66.

31. Stott B, Lavender P, Lehmann S, et al. Human IL-31 is induced by IL-4 and promotes Th2-driven inflammation. J Allergy Clin Immunol 2013; 132: 446-54.

32. Oetjen LK, Mack MR, Feng J, et al. Sensory neurons co-opt classical immune signaling pathways to mediate chronic itch. Cell 2017; 171: 217-28. 